Trabajos Originales

\title{
Religiosidad juvenil y su asociación a comportamientos sexuales en adolescentes consultantes en un centro de salud sexual y reproductiva en la Región Metropolitana
}

\author{
Electra González A. ${ }^{1, a}$, Temistocles Molina G. ${ }^{1, b}$
}

${ }^{1}$ Centro De Medicina Reproductiva y Desarrollo Integral del Adolescente (CEMERA), Facultad de Medicina, Universidad de Chile

a.Asistente Social, Master in Population Research

b.Estadistico, Magister en Bioestadistica

\section{RESUMEN}

Antecedentes: La evidencia muestra que uno de los factores fuertemente asociado con la postergación de la actividad sexual, es la afiliación o membrecía a religiones judeo-cristianas, con códigos estrictos de conductas. Objetivo: analizar la asociación entre religiosidad y comportamientos sexuales en adolescentes. Método: Estudio transversal analítico en una población adolescente. Se compararon adolescentes católicas, evangélicas y sin religión. Se analizó la asociación entre afiliación y asistencia a servicios religiosos y edad de inicio de la actividad sexual, uso de condón y número de parejas sexuales. Resultados: Se entrevistaron 5.231 adolescentes (mediana de edad 16 años, 91,6\% mujeres, 97,3\% estudiantes). Las adolescentes que se declaran evangélicas, dejan de asistir a los servicios religiosos en mayor proporción que las adolescentes católicas al iniciar actividad sexual. La afiliación religiosa, sea católica o evangélica, retarda el inicio de la actividad sexual y disminuye el riesgo de tener más de una pareja sexual, comparadas con aquellas sin religión. Pero la afiliación religiosa, sea católica o evangélica, aumenta la posibilidad del no uso de condón, en comparación con aquellas sin religión. Conclusión: La afiliación religiosa influye en los comportamientos sexuales en adolescentes, disminuyendo el riesgo en el inicio sexual y en el número de parejas sexuales, aumenta la posibilidad del no uso de condón pero no influye en el uso de anticonceptivos orales.

PALABRAS CLAVE: Afiliación religiosa, asistencia a servicios religiosos, inicio sexual, uso condón, numero parejas sexuales

\section{SUMMARY}

Background: Evidence shows that one of the factors associated with the postponement of sexual activity is membership or affiliation to Judeo-Christian religions, with strict codes of behavior. Objective: To analyze the association between religiosity and sexual behaviors in adolescents. Method: Analytical cross-sectional study conducted in a population of adolescents. Were compared catholic, evangelical and without religion. Association between affiliation and assistance religious services and age sexual initiation, condom use and number of sexual partners were analyzed. Results: In total, 5.231 adolescents (median age 16 years, 91.6\% female, 97.3\% students,), were interviewed. Adolescents who declare to be evangelicals cease to attend religious services in greater proportion than Catholic adolescents when initiating sexual activity. Religious affiliation, whether Catholic or evangelical, delay the onset of sexual activity and decreases the risk of having more than one sexual partner, compared with those without religion, but religious affiliation, whether Catholic or evangelical, increases the risk of non-use of condoms, compared to those without religion. Conclusion: Religious affiliation influences sexual 
behaviors in adolescents, decreasing the risk in the age of sexual debut and in the number of sexual partners but increasing the risk in the non-use of the condom but does not influence the pill use.

KEYWORDS: Religious affiliation, Religious services, Sexual initiation, Condom use, Number of sexual partners

\section{INTRODUCCIÓN}

La actividad sexual adolescentes está siendo cada vez más, objeto de preocupación de las políticas sanitarias y sociales en nuestro país. La actividad sexual temprana se asocia fuertemente a embarazos no deseados, adquisición de infecciones de transmisión sexual (ITS), entre otros. (1). El uso correcto del condón puede ser una solución efectiva para la prevención de ITS y en especial HIV-SIDA, pero, los estudios muestran que a menor edad de inicio sexual, es menor el uso del condón o método anticonceptivo (Mac), (1-5). Debido a lo anterior, vemos que la salud y el desarrollo socioeconómico de los jóvenes está siendo cada vez más afectado negativamente. Por esto, es que quienes toman decisiones en las políticas públicas, necesitan enfatizar aquellos factores que retrasan el inicio de la actividad sexual y favorecen el uso del preservativo y de método anticonceptivos en adolescentes que han iniciado actividad sexual (4-7).

La evidencia empírica muestra que uno de los factores que se asocia fuertemente a la postergación de la actividad sexual, es la religiosidad o afiliación a religiones judeo-cristianas, con códigos estrictos de conductas. Diversos estudios muestran que aquellas religiones que pueden influir en los comportamientos sexuales de sus feligreses, pueden retardar el inicio de la actividad sexual y pueden reducir la actividad sexual de estos, en especial en las mujeres (2,8-12). Dada esta asociación, se podría pensar que tal influencia se puede extender a otras prácticas sexuales, como número de parejas sexuales (13-20) y el uso del condón o Mac. Sin embargo, los resultados de algunos estudios resultan inconsistentes para la población adolescente en relación al uso de Mac o condón (15). Un estudio realizado en Porto Alegre muestra que no hubo diferencias significativas en el uso del condón en relación a la religiosidad, en una población de 12 a 14 años, sin embargo, los hombres lo usaron más que las mujeres para evitar ITS que para evitar embarazo (16). Un estudio realizado en México muestra que los jóvenes católicos practicantes y los evangélicos tienen menor riesgo de inicio su vida sexual que los católicos nominales y que los evangélicos tienen menores posibilidades de uso de condón en la primera relación sexual (21). Finalmente en Chile, un estudio mostró que las adolescentes que no tenían afiliación religiosa tuvieron un riesgo de $40 \%$ de abandonar el método anticonceptivo que estaban usando (5).

Según el censo de 2012, en Chile, el 70\% de la población se adscribe a la religión católica, el $15,1 \%$ a la evangélica, $4,4 \%$ a otra religión o credo y solo el $8,3 \%$ se declara sin religión. (22). Mientras que la $8^{\circ}$ Encuesta de la Juventud 2015, en la población adolescente entre 15 y 19 años, el $38,5 \%$ se declara católico, el 15,5 evangélico y el $46 \%$ sin religión (23).

Si bien todavía la mayoría de la población general y un importante porcentaje de la población adolescente se adscriben a la religión católica, la mayoría solo lo es nominalmente, es decir, no practican estrictamente todos los ritos y normas del catolicismo

En contraste con la baja participación de los católicos, las religiones evangélicas o protestantes, suelen requerir de sus fieles una decisión personal y un mayor apego a las prácticas religiosas. Se espera, por ejemplo, que las personas que se convierten a las religiones evangélicas abandonen el alcoholismo, y toda clase adicciones como tabaco, drogas, juegos de azar. Además se exige a los jóvenes abstinencia sexual antes del matrimonio y a los esposos fidelidad marital. Los fieles además son motivados a llevar una vida ejemplar como testimonio de conversión y para que sirvan de modelo de ética cristiana a la gente que los rodea. Para lograrlo, se organizan en congregaciones pequeñas que facilitan el apoyo y la interacción social así como la vigilancia de los comportamientos esperados (23-30).

En este mismo sentido, los católicos practicantes o activos, que se organizan en capillas, parroquias o grupos en familias, permiten la interacción social más estrecha que favorece el control social. (30). Así pueden acatar los diversos documentos o encíclicas de la iglesia católica que señalan su desacuerdo con el ejercicio de la sexualidad fuera del matrimonio, con el uso de 
anticonceptivos y muestran una señal represiva del sexo y del placer. (32). La religión es un agente primario de socialización en adolescentes, y la actividad sexual es una esfera del comportamiento humano de alta importancia en su aplicabilidad religiosa. (9). La religión, especialmente la evangélica, funciona animando a los jóvenes a impedir acciones que puedan llevarlos a comportamientos de riesgo. De acuerdo a ciertos autores, la influencia de la religión evangélica o protestante, ha sido vista frecuentemente como una fuerza inhibidora, la cual puede contribuir a posponer, reducir o restringir ciertos comportamientos, tales como, actividad sexual prematrimonial $(9,22$, . En Chile no se ha documentado que exista una vinculación entre religiosidad (33) y comportamientos sexuales en adolescentes.

El presente estudio tiene como objetivo analizar la asociación entre la religiosidad y comportamientos sexuales en adolescentes.

\section{PACIENTES Y MÉTODO}

Diseño de estudio transversal analítico que analizó la asociación entre la religiosidad considerando las dos más frecuentes medidas utilizadas como es la afiliación religiosa y asistencia a servicios religioso (33) y tres aspectos del comportamiento sexual adolescente: edad de inicio sexual, uso de condón y número de parejas sexuales. La población del estudio fueron adolescentes de ambos sexos que iniciaron actividad sexual y que consultaron en CEMERA durante el periodo 20002012. El estudio obtuvo la información desde una base de datos creada, para propósitos de investigación por la investigadora principal. Al ingreso a CEMERA a todos los adolescentes se les explicó la modalidad de atención y se les solicitó permiso explícito para utilizar los antecedentes que quedaron registrados en la entrevista para fines de investigación, asegurando el resguardo de la confidencialidad y anonimato de los datos. Lo anterior fue formalizado mediante firma del consentimiento informado especialmente diseñado para estos efectos.

\section{Análisis de datos}

\section{Variable resultado}

\section{Comportamientos sexuales}

Como indicador de comportamiento sexual se incluyó el Uso de condón u otro Mac al inicio de actividad sexual, se codificó como: $\mathrm{no}=1, \mathrm{si}=0$. Número de parejas sexuales se dicotomizó como: una pareja=0, dos y más $=1$ e inicio de actividad sexual en menores de 15 años.

\section{Variables independientes Variables personales}

Afiliación religiosa: Se hicieron variables dummy: Católica, Evangélica o protestante, categoría de referencia $=$ Sin religión

Asistencia actual a los servicios religiosos: Se hicieron variables dummy: nunca, ocasional, categoría de referencia=Semanal

Edad a la entrevista (años cumplidos)

Sexo: Codificada como: mujer $=0$, hombre $=1$.

Actividad: Codificada como: estudia $=0$, no estudia $=1$

Escolaridad: Se hicieron variables dummy: básica, media, categoría de referencia=Superior

Promedio de notas.

Planes futuros: Se hicieron variables dummy: solo enseñanza media, técnico, categoría de referencia=Superior

Participación social: Codificada como: participa $=0$, no participa $=1$.

\section{Variables familiares}

Filiación matrimonial: Codificada como: matrimonial $=0$, no matrimonial $=1$.

Número de hijos de la familia: Se hicieron variables dummy: dos, tres y cuatro, categoría de referencia $=$ uno

Nivel socioeconómico: Se hicieron variables dummy: medio, bajo, categoría de referencia=alto. (Usando la escala modificada de Graffar adaptada a Chile) (34).

\section{Análisis Estadístico}

Para medir la asociación entre las variables: afiliación religiosa, personales, familiares y comportamientos sexuales, se utilizó las pruebas estadísticas Chicuadrado, $t$ de Student, Test de Mann-Whitney, Análisis de Varianza (ANOVA) con post hoc Scheffe y Kruskal-Wallis. Para analizar la relación entre las variables dependientes de comportamiento sexual: uso de MAC, número de parejas sexuales y como variables independientes, afiliación religiosa, variables personales y familiares, se utilizó regresión logística múltiple. En los dos modelos se verificó interacción y confusión de las variables en estudio. La estrategia para seleccionar las variables en los modelos fue el método de "Backward" Se verificó la bondad de ajuste de los modelo mediante la prueba de "Hosmer y Lemeshow". Se utilizó el software estadístico STATA 12.0 (35). 
El estudio fue aprobado por el Comité de Ética de la Investigación en Seres Humanos de la Facultad de Medicina de la Universidad de Chile.

\section{RESULTADOS}

Los resultados corresponden a 5.231 adolescentes de ambos sexos entre 10 y 19 años, el $91,6 \%$ son mujeres, el $97,3 \%$ son estudiantes. La mediana de la edad a la entrevista fue de 16 años. El 87,5\% cursaba enseñanza media. La mediana de la edad de inicio de la actividad sexual fue de 15 años. El 61,2\% se definió católica, el 19,9\%, evangélica y 18,9\% sin religión. En relación a frecuencia a los servicios religiosos el $28,3 \%$ asiste a lo menos una vez a la semana, 33,8\% asiste una vez al mes y $37,9 \%$ no asiste nunca o casi nunca.

En las adolescentes católicas, el 27,9\% asiste semanalmente a los servicios religiosos, el $38,2 \%$ ocasionalmente y $33,9 \%$ nunca. En las adolescentes evangélicas, el $29,4 \%$ asiste semanalmente a los servicios religiosos, $20,8 \%$ ocasionalmente y $49,9 \%$, no asiste nunca o casi nunca. (No mostrado en tabla). En adolescentes católicas, la edad de inicio de la actividad sexual fue 15,4 años, en las evangélicas fue 15,3 años y en las sin religión, 15,1 años, mostrando diferencias significativas las con religión versus sin religión $(p=0,001)$. La asistencia a servicios religiosos no mostró diferencias entre católicas y evangélicas. (No mostrado en tabla).

En adolescentes menores o iguales de 15 años sin inicio de actividad sexual y católica el $33 \%$ nunca asiste a servicio religioso y en las adolescentes evangélicas, el $31 \%$, no asiste nunca. Mientras que en las que iniciaron actividad sexual, las católicas en un $34,4 \%$ nunca asiste y las con religión evangélica, el 53,4\%. (Tabla I)

Las adolescentes que se definen sin religión presentan un mayor porcentaje de uso de MAC $(64,9 \%)$, sin embargo, esta diferencia no es estadísticamente significativa. No hay diferencia en el uso de ACO en aquellas que se declaran o no con religión (Tabla II). Las adolescentes que se definieron sin religión, en un $35,5 \%$ tienen dos o más parejas sexuales cuando se comparan con las adolescentes católicas y evangélicas, $26 \%$. Lo que puede indicar que la religión si puede influir en el número de parejas sexuales que tengan las adolescentes. En cuanto a la asistencia a los servicios religiosos en la medida que la frecuencia disminuye, aumenta la frecuencia de quienes tienen dos parejas o más (Tabla III).
En el $1^{\circ}$ modelo de Regresión Logística Múltiple. Para Uso de condón resultaron significativas las siguientes variables: En adolescentes con Religión Evangélica, se incrementó el riesgo en un 20\% de no usar condón o Mac (OR: 1,20; IC95\%: 1,01 - 1,43) respecto a no tener religión. Edad, por cada año más de edad que tenga el adolescente, disminuye el riesgo en un 11,1\% (OR: 0,90; IC95\%: 0,85 - 0,95). Escolaridad básica, incrementa el riesgo en 1,46 veces (OR: 2,46; IC95\%: 1,25 - 4,85). Promedio de notas, por cada punto más en el promedio de notas que tenga el adolescente, disminuye el riesgo en un 47,1\% (OR: 0,68; IC\%:0,59 - 0,77). Planes futuros solo terminar enseñanza media, incrementa el riesgo en un $21 \%$ (OR: 1,$21 ; 1,04-1,41)) .(p<0,05)$ controlado por el resto de las variables (Tabla IV).

En el $2^{\circ}$ Modelo. Para Número de parejas sexuales resultaron significativas las siguientes variables: En adolescentes con Religión católica o Evangélica, disminuye el riesgo en un $61,3 \%$ y $53,8 \%$ tener más de una pareja sexual (OR: 0,62; IC95\%: 0,50-0,78) y (OR: 0,65; IC95\%: 0,54-0,77) respecto a no tener religión. Sexo masculino, incrementa el riesgo 1,01 veces (OR: 2,01; IC95\%: 1,59-2,55). Edad, por cada año más de edad que tenga el adolescente, aumente el riesgo en un 28\% (OR: 1,28; IC95\%: 1,20 - 1,37). Escolaridad básica incrementa el riesgo en 1,07 veces (OR: 2,07; IC95\%:1,07 - 4,0) respecto de tener educación superior. Promedio de notas, por cada punto más en el promedio de notas que tenga el adolescente, disminuye el riesgo en un 51,5\% (OR: 0,66; IC95\%: 0,57 - 0,76). Tamaño familia: Dos, Tres, Cuatro y más hijos, disminuye el riesgo un $44,9 \%, 44,9 \%$ y $53,8 \%$ (OR: 0,69; IC95\%:0,52 - 091), (OR: 0,69; IC95\%: 0,53 - 091) y (OR: 0,65; IC95\%: $0,50-0,86)$ respecto de la familia de un hijo. $(p<0,05)$ controlado por el resto de las variables (Tabla V).

\section{DISCUSIÓN}

Los resultados de este estudio muestran que la población adolescente que se definió católica es menor a la indicada en el censo de 2012 para la población general, $61,2 \%$ versus $70 \%$, pero es mayor a la reportada por la $8^{\circ}$ Encuesta de la Juventud, $38,5 \%$. El porcentaje que reportó afiliación a iglesias evangélicas o protestante fue similar a la reportada en el censo, $19,9 \%$ versus $19,5 \%$, pero mayor a la reportada por la Encuesta INJUV, donde el porcentaje fue de $15,5 \%$. Por otro lado, el porcentaje de quienes se definieron sin religión es mayor que la población 
general, $18,9 \%$ versus $8,3 \%$ pero discordante a la reportada por la Encuesta INJUV que alcanzó un 46\% para adolescentes entre 15 y 19 años.

En el caso de este estudio y el estudio del Injuv, el aumento en el grupo sin religión, quizá se deba a la disminución de quienes estaban afiliados a la iglesia católica, pero no, de quienes se declaran evangélicos. Esto puede indicar que la religión, especialmente, la evangélica, puede ser un factor importante en la vida de estos adolescentes.

En relación a la asistencia actual a los servicios religiosos, se observa que casi la mitad de las adolescentes evangélicas no están asistiendo actualmente a los servicios religiosos, porcentaje mayor que en las católicas. Una adolescente evangélica de 16 años reportó "Yo asistía todos los domingos a la Escuela Bíblica Dominical con mi abuelita, pero cuando cumplí 12 años no quise ir mas...". La entrada de sus feligreses a la adolescencia, puede ser un punto crítico para muchas iglesias evangélicas, es la etapa donde los jóvenes empiezan a explorar en conductas que pudieran ser reprobadas por la iglesia, como el inicio de las relaciones románticas, y otros comportamientos como consumo de cigarrillos, consumo de alcohol, los adolescentes evangélicos dejan de asistir a las actividades de la iglesia antes de ser descubiertos y tener que enfrentarse a las sanciones (20).

Las adolescentes católicas o evangélicas, inician actividad sexual un poco más tardía que las que declaran no tener religión, lo que es concordante con lo mostrado en la literatura que indica que la afiliación religiosa retarda el inicio de la actividad sexual (811,21).

En las adolescentes menores o iguales a 15 años, sin inicio de actividad sexual, los porcentajes de las que nunca asisten a servicios religiosos son muy similares, entre católicas y evangélicas. Pero en aquellas, que ya iniciaron actividad sexual, aumenta de manera importante el número de adolescentes evangélicas que no asisten a servicios religiosos, 53,4\%, en comparación con las católicas, 34,4\%. Esto puede indicar que las adolescentes evangélicas son más temerosas que las católicas de enfrentar las sanciones morales por la transgresión en la que están incurriendo.

Este estudio muestra que la afiliación religiosa, sea católica o evangélica, aumenta el riesgo de no uso de condón pero no influye en el uso de $A C O$, en la población adolescente estudiada. Lo que es concordante con lo encontrado en otros estudios, (1720) pero no concordante con los datos del INJUV que indica que $78,1 \%$ de los adolescentes católicos usa MAC versus el $71,5 \%$ de los adolescentes sin religión y el $70,2 \%$ de los evangélico (23).

En nuestro estudio podría ser explicado porque en ambas religiones se desincentiva el uso de métodos anticonceptivos, ya que la actividad sexual debería ocurrir siempre dentro del matrimonio, cuyo fin sería la procreación.

La afiliación religiosa, sea católica o evangélica, disminuye el riesgo de tener más de una pareja sexual. Concordante con la mayoría de los estudios incluso con los datos del INJUV que indica que un $40,8 \%$ de los que se definieron sin religión ha tenido dos o más parejas sexuales, mientras que los que declaran ser católicos un 34,5\% y evangélicas 31, 5\% (23).

Se postula entonces que, la religión, especialmente la evangélica, puede jugar un rol importante en la toma de decisiones en adolescentes en la esfera del comportamiento sexual. Esto, a través de tres factores: aspectos de orden moral, (indica lo que es bueno o malo, lo que correcto o incorrecto), sanciones religiosas (aquellos que han transgredidos las normas, son llamados a cumplir ciertas disciplinas, por ejemplo, un periodo de ausencia en la participación de los servicios religiosos, para que puedan reflexionar y arrepentirse de su conducta transgresora, hasta la expulsión de la congregación) y consecuencias por la transgresión (la violación de las normas religiosas puede generar consecuencias psicológicas, evocar sentimientos de culpa, vergüenza pública o la expectativa de castigo divino).

Pero también, la religión evangélica puede influir fuertemente, en forma indirecta, en las vidas de los jóvenes y en sus comportamientos, aumentando sus competencias en destrezas y conocimientos que contribuyen a afianzar su bienestar y mejorar sus oportunidades en la vida. En la mayoría de las congregaciones evangélicas, los niños y adolescentes pueden recibir educación bíblica y musical, pueden aprender historia, geografía y tradiciones religiosas. Participar en conjuntos corales, aprenden a tocar diversos instrumentos musicales. Se estimula desde muy pequeño, la lectura, hablar, a opinar sobre diversos temas, aprenden a coordinar talleres religiosos, asisten a programas tutoriales. Todo lo anterior, afecta positivamente su aprendizaje escolar y mejora su autoestima $(9,30)$, y puede lograr que los adolescentes desarrollen más altas aspiraciones educacionales y profesionales, como consecuencia de ello, pueden querer impedir o posponer ciertos comportamientos sexuales, tales como actividad 
sexual prematrimonial, lo cual podría tener consecuencias y restricción para mejores oportunidades de vida (28).

\section{CONCLUSION}

De acuerdo a estos resultados, la afiliación religiosa influye en los comportamientos sexuales en adolescentes, siendo un factor protector para el inicio de la actividad sexual y el número de parejas sexuales, aumentando la posibilidad del no uso de condón pero, no influye en el uso de ACO.

\section{REFERENCIAS}

1. Lammers $C$, Irelans $M$, Resnick $M$, Blum $R$. Influences on adolescents 'decision to postpone onset of sexual intercourse: a survival analysis of virginity among youth aged 13 to 18 years, J Adolescent Health, 26 (1):42-48, 2000.

2. González E. Molina T. Montero A. Martínez V. Factores asociados al inicio sexual en adolescentes de ambos sexos de nivel socioeconómico medio-bajo de la Región Metropolitana. Rev Chil Obstet Ginecol . 2013; 78(1):4-13.

3. Cunningham W, McGinnis L, García R, Tesliuc C, Verner D. Youth at risk in Latin American and the Caribbean: understanding the causes, realizing the potential, World Bank Publications, Washington D.C.2008.

4. González E. Caba F. Molina T. Sandoval J. Meneses R. Factores personales asociados al uso de anticonceptivos en adolescentes mujeres solteras sexualmente activas. Rev Soc Gin Infantil y de la Adolescencia. 2003; 10:62-9.

5. González E. Molina T. Montero A. Martínez V. Molina R. Factores asociados a la continuidad del uso anticonceptivo en adolescentes solteras nulíparas. Rev Med Chile. 2009; 137:1229-1234.

6. Breinbauer C, Maddaleno M. Youth: Choices and Change. Promoting Healthy Behaviors in Adolescents, Pan American Health Organization, Washington D, C. 2005.

7. Burdiles P. Santander S. Situación actual del embarazo adolescente en Chile. Programa Nacional de Salud Integral Adolescentes y Jóvenes. Ministerio de Salud. Chile.
8. Addai I, Religious affiliation and sexual initiation among Ghanaian women, Review of Religious Research. 2000, 41(3):328-43.

9. De Andrade Verona A. Explanations for religious influence on adolescent sexual behavior in Brasil: direct and indirect effects. R Bras Est Pop. 2011, 28 (1):187-201.

10. Hardy S, Raffaelli M. Adolescent religiosity and sexuality: an investigation of reciprocal influences. J Adolesc. 2003; 26:731-739

11. Thornton A, Camburu D. Religious participation and adolescent sexual behavior and attitudes. J Marriage and Family. 1989; 51 (3):641-653

12. Zalezki E, Schiaffino K, Religiosity and sexual risktaking behavior during the transition to college, Journal of Adolescence, 2000, 23(2):223-27.

13. Manlove J, Ryan S, Franzetta K. Patterns of contraceptive use within teenagers 'first sexual relationships. Perspective on Sexual and Reproductive Health, 2003, 35(6):246-55.

14. Bruckner $H$, Bearman $P$. After the promise: The STD Consequences of adolescent virginity pledges. J Adolescent Health, 2005, 36(4):271-78.

15. Dunne M, Edwards R, Lucke J, Donald M, Raphael $\mathrm{B}$, Religiosity, sexual intercourse and condom use among university students, Australian J Public Health, 1994, 18(3):339-41.

16. Cerqueira-Santos E, Koller S, Wlicox B. Condom use, contraceptive methods, and religiosity among youths of low socioeconomic level, Spanish J Psychology, 2008, 11(1):94-102.

17. Rostosky SS, Wilcox BL, Wright ML, Randall BA. The impact of religiosity on adolescent sexual behavior: A review of the evidence. J Adolescent Research, 2004; 19(6):677-697

18. Wilcox BL, Rostosky SS, Randall BA, Wright ML. Reason for hope: A review of research on adolescent religiosity and sexual behavior. In: B D Whitehead, BL Wilcox and SS Rostosky (Eds). Keeping the Faith: The role of religion and faith communities in preventing teen pregnancy. Washington DC: The National Campaign to Prevent Teen Pregnancy, 2001, pp 31-82.

19. Vasilenko S, Duntzee C, Zheng Y, Lefkowitz E, Testing two process models of religiosity and sexual behavior, J Adolesc, 2013, 36(4):667-673. 
20. LeJeune B, Zimet G, Azzouz F, Fortenberry J D, Aalsma M, Religiosity and sexual involvement within adolescent romantic couples, J Relig Health, 2013, 52(3):804-816

21. Vargas E, Martínez G, Potter J. Religión e iniciación sexual premarital en México. Revista Latinoamericana de Población, 2010; 4(1):7-30.

22. INE, Censo, 2012.

23. $8^{\circ}$ Encuesta Nacional de la Juventud 2015. INJUV. Ministerio de Desarrollo Social. Gobierno de Chile. Análisis propio.

24. Miller L, Gur M. Religiousness and sexual responsibility in adolescent girls. J Adolescent Health; 2002; 31: 401-406.

25. Nonnemaker M. J, McNeely C.A. Blum R. Public and private domains of religiosity and adolescent health risk behaviors: evidence from the National Longitudinal Study of Adolescent Health. Social Science \& Medicine, 2003, 57:2049-2054.

26. Powers D, Ellison C. Conservative Protestantism and Church attendance effects on Teen pregnancy and pregnancy outcomes. Department of Sociology and Population Research Center, University of Texas at Austin, Paper presented to the March 31-april 2, 2005, Annual Meeting of the Population Association of America, Philadelphia.

27. Mundigo A. Religión y salud reproductiva: encrucijadas y conflictos. II Reunión de investigación sobre embarazo no deseado y aborto inseguro. Desafíos de salud publica en América Latina y el Caribe. Ciudad de México, 2005.
28. Smith C. Religious participation and network closure among American adolescents. $J$ for the Scientific Study of Religion. 2003. 42(2):259-267.

29. Gold M.A., Sheftel A.V., Chiapetta L. Young A.J. Zuckoff A. DiClemente C.C., Primack B.A. Associations between religiosity and sexual and contraceptive behaviors. J Pediatr Adolesc Gynecol. 2010. 23(5):290-297.

30. Vásquez $F$, La praxis de la fe evangélica en la sociedad, Graffylia, 2009, 1(2):113-123.

31. Castro C. Transformarse desde adentro: la religión católica. Revista Transición, Debate y Propuesta en Veracruz, 2002, 42:3-5.

32. Hernández J. Sexualidad y afectividad en el religioso católico. Estudios sobre las Culturas Contemporáneas, 2002, VIII, (015):57-88.

33. Williams D, The measurement of religion in epidemiologic studies. In: Jeffrey S. Levin (Ed) Religion in Aging and Health: Theoretical Foundations and Methodological Frontiers. Thousand Oaks, CA: Sage Publications, 1994.pp 125-147.

34. Alvarez M, Muzzo S, Ivanovic D. Escala para medición del nivel socioeconómico en el área de salud. Rev Med Chile, 1985;113:243-9

35. Hosmer DW, Lemeshow S. Applied Logistic Regression, 2nd Edition; New York: John Wiley \& Sons. INC, 2000 
Tabla I

DISTRIBUCION DE ASISTENCIA A SERVICIO RELIGIOSO SEGÚN RELIGION E INICIO O NO INICIO DE ACTIVIDAD SEXUAL EN ADOLESCENTES MENORES O IGUALES A 15 AÑOS

\begin{tabular}{|l|c|c|c|c|c|}
\hline \multirow{2}{*}{$\begin{array}{c}\text { Inicio de } \\
\text { actividad } \\
\text { sexual }\end{array}$} & Religión & $\begin{array}{c}\text { Semanal } \\
\mathbf{n}(\%)\end{array}$ & $\begin{array}{c}|c| \\
\text { Ocasional } \\
\mathbf{n}(\%)\end{array}$ & $\begin{array}{c}\text { Nunca } \\
\mathbf{n}(\%)\end{array}$ & $\begin{array}{c}\text { Total } \\
\mathbf{n}(\%)\end{array}$ \\
\hline \multirow{2}{*}{$\begin{array}{l}\text { Sin inicio de } \\
\text { actividad } \\
\text { sexual }\end{array}$} & Católica & $105(31,3)$ & $119(35,4)$ & $112(33,3)$ & $336(100)$ \\
\cline { 2 - 6 } & Otra religión & $39(46,4)$ & $19(22,6)$ & $26(31,0)$ & $84(100)$ \\
\hline $\begin{array}{l}\text { Con inicio de } \\
\text { actividad } \\
\text { sexual }\end{array}$ & Católica & $164(25,6)$ & $257(40,0)$ & $221(34,4)$ & $642(100)$ \\
\hline & Otra religión & $51(27,0)$ & $37(19,6)$ & $101(53,4)$ & $189(100)$ \\
\hline
\end{tabular}

Tabla II

\section{DISTRIBUCION DE USO DE MAC SEGÚN RELIGION, ASISTENCIA A SERVICIOS RELIGIOSOS Y VARIABLES SOCIODEMOGRAFICAS}

\begin{tabular}{|c|c|c|c|c|}
\hline & \multicolumn{2}{|c|}{ Uso de MAC'1 } & \multirow{2}{*}{$\begin{array}{l}\text { Total } \\
\mathrm{n}(\%)\end{array}$} & \multirow[b]{2}{*}{$\mathbf{p}$} \\
\hline & $\begin{array}{c}\mathbf{S i} \\
\mathbf{n}(\%)\end{array}$ & $\begin{array}{c}\text { No } \\
n(\%)\end{array}$ & & \\
\hline \multicolumn{4}{|l|}{ Religión } & \multirow{4}{*}{0,33} \\
\hline Católica & $1534(62,1)$ & $935(37,9)$ & $2469(100)$ & \\
\hline Evangélica & $493(61,9)$ & $303(38,1)$ & $796(100)$ & \\
\hline Sin religión & $515(64,9)$ & $278(35,1)$ & $793(100)$ & \\
\hline \multicolumn{4}{|c|}{$\begin{array}{l}\text { Asistencia a servicios } \\
\text { religiosos }\end{array}$} & \multirow{4}{*}{0,69} \\
\hline Semanal & $536(60,8)$ & $345(39,2)$ & $881(100)$ & \\
\hline Ocasional & $689(62,6)$ & $411(37,4)$ & $1100(100)$ & \\
\hline Nunca & $792(62,3)$ & $480(37,7)$ & $1272(100)$ & \\
\hline \multicolumn{4}{|l|}{ Sexo } & \multirow{3}{*}{0,41} \\
\hline Femenino & $2326(62,3)$ & $1405(37,7)$ & $3731(100)$ & \\
\hline Masculino & $240(64,5)$ & $132(35,5)$ & $372(100)$ & \\
\hline \multicolumn{4}{|l|}{ Actividad } & \multirow{3}{*}{0,01} \\
\hline Estudiante & $2499(62,9)$ & $1475(37,1)$ & $3974(100)$ & \\
\hline No estudiante & $67(51,9)$ & $62(48,1)$ & $129(100)$ & \\
\hline \multicolumn{4}{|l|}{ Escolaridad } & \multirow{4}{*}{0,001} \\
\hline Basica & $146(44,4)$ & $183(55.6)$ & $329(100)$ & \\
\hline Media & $2364(63,9)$ & $1338(36,1)$ & $3702(100)$ & \\
\hline Superior & $54(79,4)$ & $14(20,6)$ & $68(100)$ & \\
\hline \multicolumn{4}{|l|}{ Planes futuros } & \multirow{4}{*}{0,001} \\
\hline Solo EM & $628(57,0)$ & $473(43,0)$ & $1101(100)$ & \\
\hline Técnico & $194(62,4)$ & $117(37,6)$ & $311(100)$ & \\
\hline Superior & $1731(65,1)$ & $926(34,9)$ & $2657(100)$ & \\
\hline
\end{tabular}




\begin{tabular}{|c|c|c|c|c|}
\hline Participación social & & & & \multirow[b]{3}{*}{0,02} \\
\hline $\mathrm{Si}$ & $1167(64,7)$ & $637(35,3)$ & 1804(100) & \\
\hline No & $1380(61.0)$ & $881(39,0)$ & $2261(100)$ & \\
\hline \multicolumn{4}{|l|}{ Filiación Matrimonial } & \multirow{3}{*}{0,60} \\
\hline $\mathrm{Si}$ & $1910(62,9)$ & $1128(37,1)$ & $3038(100)$ & \\
\hline No & $648(62,0)$ & $398(38,0)$ & $1046(100)$ & \\
\hline \multicolumn{4}{|c|}{ Número de hijos de la familia } & \multirow{5}{*}{0,05} \\
\hline Uno & $215(66,0)$ & $111(34,0)$ & $326(100)$ & \\
\hline Dos & $756(64,0)$ & $425(36,0)$ & $1181(100)$ & \\
\hline Tres & $836(63,5)$ & $480(36,5)$ & $1316(100)$ & \\
\hline Cuatro y más & $756(59,6)$ & $513(40,4)$ & $1269(100)$ & \\
\hline \multicolumn{4}{|l|}{ NSE } & \multirow{4}{*}{0,23} \\
\hline Alto & $56(70,9)$ & $23(29,1)$ & $79(100)$ & \\
\hline Medio & $670(66,0)$ & $345(34,0)$ & $1015(100)$ & \\
\hline Bajo & $449(63,0)$ & $264(37,0)$ & $713(100)$ & \\
\hline $\begin{array}{l}\text { Edad } \\
\text { Promedio (DE) } \\
\text { Mediana }\end{array}$ & $\begin{array}{c}16,3(1,2) \\
16\end{array}$ & $\begin{array}{c}16,0(1,3) \\
16\end{array}$ & & 0,001 \\
\hline $\begin{array}{l}\text { Promedio de notas } \\
\text { Promedio (DE) } \\
\text { Mediana }\end{array}$ & $\begin{array}{c}5,5(0,5) \\
5,5\end{array}$ & $\begin{array}{c}5,4(0,5) \\
5,4\end{array}$ & & 0,001 \\
\hline
\end{tabular}


Tabla III

DISTRIBUCION DE NÚMERO DE PAREJAS SEXUALES SEGÚN RELIGION, ASISTENCIA A SERVICIOS RELIGIOSOS Y VARIABLES SOCIODEMOGRAFICAS

\begin{tabular}{|c|c|c|c|c|}
\hline & \multicolumn{2}{|c|}{$\mathrm{N}^{\circ}$ de parejas sexuales } & \multirow{2}{*}{$\begin{array}{l}\text { Total } \\
\mathrm{n}(\%)\end{array}$} & \multirow[b]{2}{*}{$\mathbf{p}$} \\
\hline & $\begin{array}{l}\text { Una } \\
\mathrm{n}(\%)\end{array}$ & $\begin{array}{c}\text { Dos y más } \\
n(\%)\end{array}$ & & \\
\hline \multicolumn{4}{|l|}{ Religión } & \multirow{4}{*}{0,001} \\
\hline Católica & $1832(74,0)$ & $643(26,0)$ & $2475(100)$ & \\
\hline Evangelica & $589(73,4)$ & $213(26,6)$ & $802(100)$ & \\
\hline Sin religión & $516(64,5)$ & $284(35,5)$ & $800(100)$ & \\
\hline \multicolumn{4}{|c|}{$\begin{array}{l}\text { Asistencia a servicios } \\
\text { religiosos }\end{array}$} & \multirow{4}{*}{0,02} \\
\hline Semanal & $678(76,4)$ & $209(23,6)$ & $887(100)$ & \\
\hline Ocasional & $826(74,6)$ & $281(25,4)$ & $1107(100)$ & \\
\hline Nunca & $906(71,3)$ & $365(28,7)$ & $1271(100)$ & \\
\hline \multicolumn{4}{|l|}{ Sexo } & \multirow{3}{*}{0,001} \\
\hline Femenino & $2766(73,8)$ & $983(26,2)$ & $3749(100)$ & \\
\hline Masculino & $195(52,4)$ & $177(47,6)$ & $372(100)$ & \\
\hline \multicolumn{4}{|l|}{ Actividad } & \multirow{3}{*}{0,001} \\
\hline Estudiante & $2895(72,5)$ & $1098(27,5)$ & $3993(100)$ & \\
\hline No estudiante & $66(51,6)$ & $62(48,4)$ & $128(100)$ & \\
\hline \multicolumn{4}{|l|}{ Escolaridad } & \multirow{4}{*}{0,87} \\
\hline Basica & $240(72,3)$ & $92(27,7)$ & $332(100)$ & \\
\hline Media & $2672(71,9)$ & $1046(28,1)$ & $3718(100)$ & \\
\hline Superior & $47(69,1)$ & $21(30,9)$ & $68(100)$ & \\
\hline \multicolumn{4}{|l|}{ Planes futuros } & \multirow{4}{*}{0,56} \\
\hline Estudiar solo EM & $795(72,0)$ & $309(28,0)$ & $1104(100)$ & \\
\hline Tecnico & $235(74,6)$ & $80(25,4)$ & $315(100)$ & \\
\hline Superior & $1913(71,7)$ & $755(28,3)$ & $2668(100)$ & \\
\hline \multicolumn{4}{|c|}{ Participación social } & \multirow{3}{*}{0,69} \\
\hline $\mathrm{Si}$ & $1300(71,7)$ & $514(28,3)$ & $1814(100)$ & \\
\hline No & $1639(72,2)$ & $630(27,8)$ & $2269(100)$ & \\
\hline \multicolumn{4}{|c|}{ Filiación Matrimonial } & \multirow{3}{*}{0,82} \\
\hline Si & $2197(72,0)$ & $854(28,0)$ & $3051(100)$ & \\
\hline No & $753(71,7)$ & $298(28,3)$ & $1051(100)$ & \\
\hline \multicolumn{4}{|c|}{$\begin{array}{l}\text { Número de hijos de la } \\
\text { familia }\end{array}$} & \multirow{5}{*}{0,07} \\
\hline Uno & $215(65,8)$ & $112(34,2)$ & $327(100)$ & \\
\hline Dos & $861(72,8)$ & $322(27,2)$ & $1183(100)$ & \\
\hline Tres & $950(71,9)$ & $372(28,1)$ & $1322(100)$ & \\
\hline Cuatro y más & $929(72,7)$ & $349(27,3)$ & $1278(100)$ & \\
\hline \multicolumn{4}{|l|}{ NSE } & \multirow{3}{*}{0,93} \\
\hline Alto & $59(72,0)$ & $23(28,0)$ & $82(100)$ & \\
\hline Medio & $713(69,9)$ & $307(30,1)$ & $1020(100)$ & \\
\hline
\end{tabular}




\begin{tabular}{|l|c|c|c|}
\hline Bajo & $504(70,2)$ & $214(29,8)$ & $718(100)$ \\
\hline $\begin{array}{l}\text { Edad } \\
\text { Promedio(DE) }\end{array}$ & $16,1(1,2)$ & $16,4(1,3)$ & 17 \\
Mediana & 16 & 0,001 \\
\hline $\begin{array}{l}\text { Promedio de notas } \\
\text { Promedio(DE) } \\
\text { Mediana }\end{array}$ & $5,5(0,5)$ & $5,4(0,5)$ & 0,001 \\
\hline
\end{tabular}

Tabla IV

\section{REGRESION LOGISTICA MULTIPLE ENTRE USO DE MAC Y RELIGION Y OTRAS VARIABLES SOCIODEMOGRAFICAS}

\begin{tabular}{|c|c|c|c|}
\hline Variable & Odds Ratio & $\mathbf{p}$ & IC (95\%) \\
\hline $\begin{array}{l}\text { Religión: } \\
\text { Católica }^{1} \\
\text { Evangelica }^{1}\end{array}$ & $\begin{array}{l}1,23 \\
1,20\end{array}$ & $\begin{array}{l}0,06 \\
0,04\end{array}$ & $\begin{array}{l}0,99-1,51 \\
1,01-1,43\end{array}$ \\
\hline Edad & 0,90 & 0,0001 & $0,85-0,95$ \\
\hline $\begin{array}{l}\text { Escolaridad: } \\
\text { Básica }^{2} \\
\text { Media }^{2}\end{array}$ & $\begin{array}{l}2,46 \\
1,57\end{array}$ & $\begin{array}{c}0,009 \\
0,14\end{array}$ & $\begin{array}{l}1,25-4,85 \\
0,86-2,88\end{array}$ \\
\hline Promedio de notas & 0,68 & 0,0001 & $0,59-0,77$ \\
\hline $\begin{array}{l}\text { Planes futuros: } \\
\text { Solo enseñanza media }{ }^{3} \\
\text { Técnico }^{3}\end{array}$ & $\begin{array}{l}1,21 \\
1,06\end{array}$ & $\begin{array}{l}0,01 \\
0,66\end{array}$ & $\begin{array}{l}1,04-1,41 \\
0,82-1,36\end{array}$ \\
\hline \multicolumn{4}{|c|}{$\begin{array}{l}\text { 1: Religión: categoría de referencia: sin religión } \\
\text { 2: Escolaridad: categoría de referencia: superior } \\
\text { 3: Planes futuros: categoría de referencia: educación superior Hosmer-Lemeshow: } p=0,12\end{array}$} \\
\hline
\end{tabular}


Tabla V

REGRESION LOGISTICA MULTIPLE ENTRE NÚMERO DE PAREJAS SEXUALES Y RELIGION Y OTRAS VARIABLES SOCIODEMOGRAFICAS

\begin{tabular}{|c|c|c|c|}
\hline Variable & Odds Ratio & $\mathbf{p}$ & IC (95\%) \\
\hline $\begin{array}{l}\text { Religión: } \\
\text { Católica }^{1} \\
\text { Evangelica }^{1}\end{array}$ & $\begin{array}{l}0,62 \\
0,65\end{array}$ & $\begin{array}{l}0,0001 \\
0,0001\end{array}$ & $\begin{array}{l}0,50-0,78 \\
0,54-0,77\end{array}$ \\
\hline $\mathrm{Sexo}^{2}$ & 2,01 & 0,0001 & $1,59-2,55$ \\
\hline Edad & 1,28 & 0,0001 & $1,20-1,37$ \\
\hline $\begin{array}{l}\text { Escolaridad: } \\
\text { Básica }^{3} \\
\text { Media }^{3}\end{array}$ & $\begin{array}{l}2,07 \\
1,57\end{array}$ & $\begin{array}{l}0,03 \\
0,11\end{array}$ & $\begin{array}{c}1,07-4,0 \\
0,90-2,75\end{array}$ \\
\hline Promedio de notas & 0,66 & 0,0001 & $0,57-0,76$ \\
\hline $\begin{array}{l}\text { Número de hijos de la } \\
\text { Dos }^{4} \\
\text { Tres }^{4} \\
\text { Cuatro y más }\end{array}$ & $\begin{array}{l}0,69 \\
0,69 \\
0,65\end{array}$ & $\begin{array}{l}0,008 \\
0,008 \\
0,002\end{array}$ & $\begin{array}{l}0,52-0,91 \\
0,53-0,91 \\
0,50-0,86\end{array}$ \\
\hline $\begin{array}{l}\text { 1: Religión: categoría de } \\
\text { 2: Sexo: categoría de re } \\
\text { 3: Escolaridad: categorí } \\
\text { 4: Número de hijos de la }\end{array}$ & ligión & mor & \\
\hline
\end{tabular}

